disability. A new disease specific measure of hand OA has been developed, termed the Australian/Canadian (AUSCAN) osteoarthritis hand index.

Objectives To make preliminary investigations of the performance of the Norwegian version of AUSCAN by examining correlations between scores of AUSCAN and other generic and disease specific health status measures.

Methods The AUSCAN is a disease specific health status measure for hand OA measuring pain (5 items), stiffness (1 item) and difficulties with daily activities ( 9 items). The instrument was translated to Norwegian according to standardised procedures. Fiftyone patients between 50 and 70 years of age with hand OA (mean (SD) age 66.1 (4.4) years, 4(8\%) males) underwent a comprehensive clinical examination including completion of several self-reported health status questionnaires. Among these were AUSCAN, AIMS2, SF-36, MHAQ, pain and fatigue on VAS. All patients had previously been referred to a rheumatology outpatient department. Internal validity was examined by Cronbach alpha, correlations with other scales by Pearson correlation coefficients. Our pre-study hypothesis was to confirm AUSCAN as a specific hand measure with strong or substantial correlations ( $\mathrm{r}$ $>0.50)$ to hand measures of similar dimensionality, and lower correlations between the AUSCAN measures and generalised measures within the same dimension of health, and low correlations between AUSCAN scales and measures capturing different dimensions.

Results The internal validity of the AUSCAN scales was satisfactory (Cronbach alpha 0.95 and 0.93 for AUSCAN physical and pain, respectively). Correlations between AUSCAN physical and stiffness/pain were $0.67 / 0.85$, between pain and stiffness 0.67 . The correlation between the physical scale/pain scale/stiffness scales of AUSCAN with AIMS2 hand and finger function was $0.70 / 0.54 / 0.33$ respectively, with grip strength ?0.55/-0.42/-0.22, MHAQ 0.49/0.37/0.39, AIMS2 physical 0.52/0.44/0.26, SF-36 physical ?0.27/-0.23/-0.10, WOMAC physical 0.37/0.43/0.33, WOMAC stiffness $0.20 / 0.27 / 0.27$, WOMAC pain $0.27 / 0.40$ / 0.28 , pain VAS $0.31 / 0.45 / 0.33$, AIMS2 pain $0.47 / 0.59 / 0.45$, SF36 pain ?0.37/-0.42/-0.24, fatigue VAS $0.34 / 0.37 / 0.11$, AIMS2 affect $0.32 / 0.36 / 0.04$, and with SF-36 mental ?0.14/-0.13/0.17, for the physical/pain/stiffness scales of AUSCAN, respectively.

Conclusion In general, our pre-study hypothesis was supported by the results. The physical dimension of AUSCAN appeared to be strongly or substantially correlated to other measures of hand function, and moderately correlated to other measures of physical disability. The pain measure was moderately correlated to other pain measures, whereas the correlation between the stiffness dimension of AUSCAN and WOMAC was rather low (0.27). These preliminary data indicate that the Norwegian version of AUSCAN is appropriate for use in patients with hand OA.

\section{SAT0078 EXPERIENCES WITH A VISCOSUPPLEMENTATION (HYALGAN) CLINIC}

C Rao, N Kumar, S Young-Min, NJ Marshall, PN Platt, ID Griffiths. Department of Rheumatology, Freeman Hospital, Newcastle Upon Tyne, England, UK

\subsection{6/annrheumdis-2001.453}

Background Osteoarthritis (OA) is the commonest condition to affect human joints. It is a major cause of pain, disability and handicap, and a difficult condition to treat. Pharmacological treatment has been limited to the use of analgesics, non-steroidal anti-inflammatory drugs and the controversial use of intra-articular steroid injections. None of these options provide a truly effective treatment. Viscosupplementation has only recently become available in the UK. Hyaluronon in synovial fluid has multiple functions in joint homeostasis. It is reduced in concentration and molecular weight in OA. Therefore viscosupplementation would not only remove pathological synovial fluid (by prior joint aspiration) but also restore the molecular weight and concentration of hyaluronan. ${ }^{1}$

Objectives We wished to evaluate viscosupplementation for OA of the knee, and to ascertain which patient groups would derive the most benefit from Hyalgan.

Methods We have an ongoing dedicated visco-supplementation clinic. Patients are assessed to ensure suitability and are also advised about weight reduction (if appropriate) and quadriceps exercises. They attend weekly for the course of 5 injections. The patients are asked to complete a WOMAC Questionnaire precourse and at 6 weeks, 3 and 6 months after treatment. This is a health status instrument for measuring clinically important patient relevant outcomes to anti-rheumatic drug therapy in patients with osteoarthritis of the hip or knee. ${ }^{2}$ No ethical approval was required.

Results Comparing pre-course WOMAC scores with all time intervals a statistically significant difference at 6 weeks (paired T test $\mathrm{p}=0.0081$ ) was found. Patients were sub-divided into 2 groups according to WOMAC scores, 1600. (Maximum score 2400 , indicating maximum severity). These groups were also compared. Results to reach statistical significance were WOMAC changes at 6 weeks and 6 months in the $>1600$ group, compared to pre-course where $\mathrm{p}=0.0053$ and $\mathrm{p}=0.023$ respectively (unpaired $\mathrm{T}$ test).

Conclusion From our study patients reported an improved WOMAC at 6 weeks. Those with score $>1600$, pre-course do better than those with scores inconclusive but do appear to offer some benefit to patients. ${ }^{3}$ As with intra-articular steroid injections in this condition, it is difficult to predict who will benefit. ${ }^{4}$ Our work would suggest that patients with higher initial scores on WOMAC questionnaire may respond better.

\section{REFERENCES}

1 Marshall KW. Intra-articular hyaluronan therapy. Curr Opin Rheumatol. 2000;12 (5):468-74

2 Bellamy N, et al. Validation study of WOMAC. J Rheumatol. 1988;15:1833-40

3 Wen DY. Intra-articular hyaluronic acid for knee osteoarthritis. Am Fam Physician 2000;62(3):565-70

4 Jones A, Doherty M. Intra-articular corticosteroids are effective in osteoarthritis but there are no clinical predictors of response. Ann Rheum Dis. 1996;55 (11):829-32

\section{SAT0079 VALIDITY OF ARTHROSCOPIC GRADING SYSTEM OF CHONDROPATY IN KNEE OSTEOARTHRITIS: CORRELATION WITH HISTOPATHOLOGICAL FINDINGS}

${ }^{1} \mathrm{C}$ Acebes, ${ }^{1} \mathrm{I}$ Palacios, ${ }^{2} \mathrm{E}$ Delgado, ${ }^{1} \mathrm{G}$ Herrero-Beaumont. ${ }^{1}$ Rheumatology, Fundación Jiménez Díaz, Madrid; ${ }^{2}$ Histopathology, Universidad Autonoma, Madrid, Spain

\subsection{6/annrheumdis-2001.454}

Background Clinical research in osteoarthritis (OA) requires objective methods able to evaluate cartilage status. Arthroscopic evaluation of the articular cartilage would be a reliable instrument for the measurement of the quantity, integrity or quality of articular cartilage in knee OA.

Objectives To correlate an arthroscopic grading system of chondropathy (CHGS) with the histopathological findings of the 